\title{
Octave-Tunable Miniature RF Resonators
}

\author{
James R. White, Christopher J. White, and Alexander H. Slocum
}

\begin{abstract}
The development and testing of a miniaturized, high- $Q$, broadly tunable resonator is described. An exemplary device, with a center frequency that is continuously tunable from 1.2 to $2.6 \mathrm{GHz}$, was tested in detail. Experimental results demonstrated a resonator $Q$ of up to 380 , and typical insertion loss of $-1.9 \mathrm{~dB}$ for a $25 \mathrm{MHz}$ 3-dB bandwidth. These resonators have been used to stabilize a broadly-tunable oscillator with phase noise of $-132 \mathrm{dBc} / \mathrm{Hz}$ at $100-\mathrm{kHz}$ offset, with a center frequency tunable from 1.2-2.6 GHz, and a tuning speed of $1 \mathrm{GHz} / \mathrm{ms}$.
\end{abstract}

Index Terms-Microelectromechanical system (MEMS), resonators, voltage controllable oscillators (VCOs).

\section{INTRODUCTION}

$\mathbf{T}$ UNABLE radio frequency devices have long relied on nonlinear elements such as ferrites [1], ferroelectrics [2], or varactors [3] to implement frequency-tuning. A central weakness of all these approaches is the nonlinearity of the tuning element, and the resulting performance degradation such as intermodulation at high power levels. For example, a $700 \mathrm{MHz}$ to $1.33 \mathrm{GHz}$ varactor-tuned filter with 3-dB insertion loss for a $16 \%$ bandwidth was demonstrated by [4], but IP3 intermodulation was poor at $17.9 \mathrm{dBm}$. A ferrite-tuned $4-40 \mathrm{GHz}$ wide bandwidth filter was demonstrated by [5], however bandwidth was not constant over the tuning range and the tuning current was $1.5 \mathrm{~A}$, indicating a large power requirement, and the use of tuning magnets suggests a large mass. Magnetically-tuned superconducting filters were demonstrated by [6], with $10-\mathrm{GHz}$ center frequency, $1 \% \mathrm{BW}$, and $13 \%$ frequency tuning, using a superconducting Yttrium barium copper oxide (YBCO) stripline and very small tuning field to vary the ferrite magnetization. This filter demonstrates IP3 intermodulation of approximately $+40 \mathrm{dBm}$ but the filter response degrades above $+10 \mathrm{dBm}$ input power, a limitation of the superconducting stripline. A three-pole Ka-band filter [7] made by the EFAB process demonstrated insertion loss of $1.74 \mathrm{~dB}$ at $29 \mathrm{GHz}$ for a 6-GHz BW, indicating an unloaded resonator $Q$ of approximately 50 .

A widely promoted alternative to the classical approach is the use of micromachined variable-capacitive elements [8]. Several groups have also demonstrated micromachined fixed-frequency cavities in the mm-wave bands, for example a two-pole bandpass filter with $3 \% \mathrm{BW}$ at $12.7 \mathrm{GHz}$ and insertion loss of $1.88 \mathrm{~dB}$

Manuscript received April 4, 2005; revised July 15, 2005. The work was supported in part by the MIT Deshpande Center for Technological Innovation. The review of this letter was arranged by Associate Editor M. Mozowski.

J. R. White and A. H. Slocum are with the Massachusetts Institute of Technology, Cambridge, MA 02139 USA (e-mail: jwhite@mit.edu).

C. J. White is with the California Institute of Technology, Pasadena, CA 91125 USA.

Digital Object Identifier 10.1109/LMWC.2005.859012

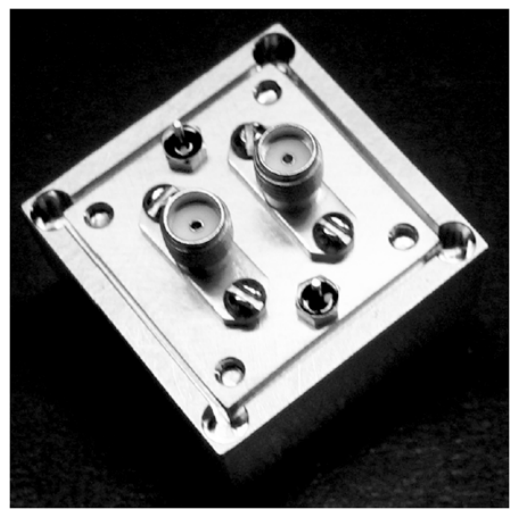

Fig. 1. Packaged $S$-band tunable resonator.

[9]. An electrostatically tuned cavity resonator at mm-wave frequencies was proposed [10] but not reduced to practice. Such an electrostatically-tuned arrangement would present a limitation on power handling due to pull-in at high radio frequency (RF) power, and intermodulation due to self-excitation around the mechanical resonant frequency of the cavity.

Resonators tuned by micromachined capacitive elements have also often fallen short of required performance metrics such as immunity to self-excitation and manufacturability, leading to a dearth of frequency agile MEMS devices in commercial use [11]. This has prompted the development of the "Octave+" miniaturized mechanically tunable resonator, shown in packaged form in Fig. 1. The resonator design was based on classical precision-mechanism design principles [12], [13], resulting in a simplified manufacturing process, and excellent mechanical and electrical performance. The resonator consists of a copper cavity loaded by a capacitive gap. The resonator is tuned using a piezo disc to deform the cavity, as shown in Fig. 1.

\section{ELECTRICAL DESIGN}

The tunable resonator is a flexible copper re-entrant cavity, whose physical dimensions are sufficiently small compared to an electrical wavelength, that there exist identifiable inductive and capacitive regions. An outer toroidal region with fixed geometry contains only the magnetic field and is therefore modeled as an inductor. An inner region with opposed parallel plates with variable separation contains the oscillating electric field and is modeled as a tunable capacitor. Thus, the device can be accurately modeled as a tunable parallel-LC circuit for all resonant frequencies, as shown in Fig. 2. In the exemplary implementation described in this letter, the input and output signals are capacitively coupled into the resonator, and the capacitive coupling elements vary in concert with the tank capacitance. 


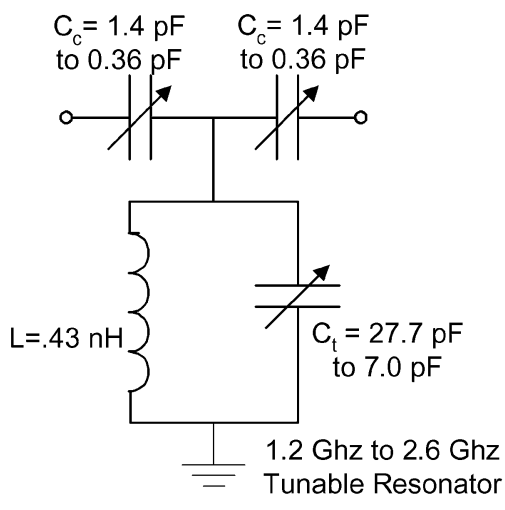

Fig. 2. Equivalent circuit model (two port $S$-band resonator).

\section{Mechanical Design}

A piezoelectric d31-mode bending unimorph actuator was designed as the tuning element. Piezoelectric actuators offer the advantage of robustness, high precision and relative simplicity when compared to, for example, electrostatic or magnetostrictive elements. Notwithstanding their advantages, piezoelectric devices suffer from well known nonlinearities such as hysteresis and creep that are readily compensated using external feedback control [14].

The $S$-band tunable resonators are assembled using conductive epoxy, although solder joints can also be employed. In operation, they are tuned by applying an electric field across a .004 in thick disk of piezoelectric material bonded with conductive epoxy ${ }^{1}$ to the top surface of the .0032 in thick electroformed copper resonator. A cross section of this arrangement is shown in Fig. 3. The electroformed parts are plated off a machined and polished aluminum mandrel, and released in caustic solution. An earlier resonator design used etched silicon [15].

Finite-element analysis (FEA) was used to predict the static deflection of the piezoelectric unimorph structure. This analysis employed a convenient analogy to thermal bimorph structures, as in [16], where the piezoelectric charge constant $d_{31}$ is analogous to the thermal expansion coefficient, and the applied electric field is analogous to temperature. This technique can be used to model low frequency actuation. An actuator response of $0.16 \mu \mathrm{m} / \mathrm{V}$ was predicted for the fabricated resonator structure, however the measured results gave a response of $.07 \mu \mathrm{m} / \mathrm{V}$. The large discrepancy has been attributed to the low shear stiffness of the adhesive layer between the resonator and the piezoelectric disc. However, the FEA was useful in predicting the optimal dimensions of the piezoelectric disks and the optimal wall thickness of the electroformed copper cavity. The initial capacitor gap was $12.8 \mu \mathrm{m}$, corresponding to a resonator center frequency of $2.30 \mathrm{GHz}$.

\section{DYNAMICS}

The dynamic response of the mechanical structure was also modeled by finite-element methods. The lowest resonant mode occurs at $5.32 \mathrm{kHz}$. The static stiffness of the resonator structure (open-loop piezo) was $7.6 \times 10^{4} \mathrm{~N} / \mathrm{m}$. A second-order lumped-

${ }^{1}$ Epotek H20E, Epotek Inc., Billerica, MA.

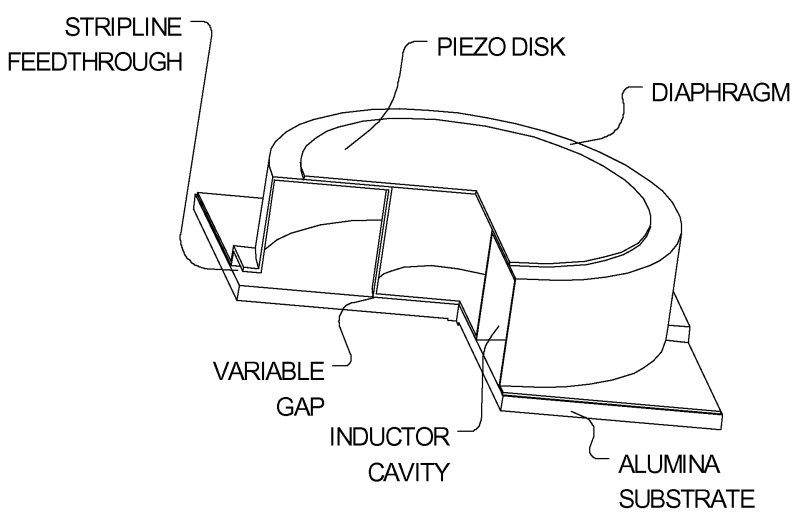

Fig. 3. Perspective view of tunable resonator.

parameter model was then constructed to model the resonator's sweep rate, settling time and sensitivity to self-excitation

$$
m_{\mathrm{eq}} \ddot{h}+b(h) \dot{h}+k h=\frac{\varepsilon_{0} A_{c} V_{\mathrm{RF}}^{2}}{\left(h_{0}-h\right)^{2}}+P_{a}(t) A_{t}+K_{p} V_{p}(t) .
$$

Here, $h$ is the displacement of the parallel-plate capacitor, $m_{\mathrm{eq}}$ is the equivalent lumped-parameter mass, $b(h)$ is the nonlinear damping coefficient, $k$ is the stiffness of the actuator (calculated from the measured resonant frequency), $\varepsilon_{0}$ is the permittivity of free space, $A_{\mathrm{c}}$ is the area of the capacitor, $\mathrm{V}_{\mathrm{RF}}$ is the the slowly-varying envelope of the RF field, since the mechanical structure acts as a low-pass filter, $h_{0}$ is the initial capacitor gap, $P_{A}(t)$ is the acoustic pressure due to microphonic disturbances, $A_{t}$ is the projected area of the resonator, $K_{P}$ is the measured response coefficient of the piezoelectric actuator, and $\mathrm{V}_{P}(t)$ is the voltage applied to the piezo diaphragm. The damping of the system is modeled as squeeze-film damping [17]. The damping coefficient $b(h)$ is given by

$$
b(h)=\frac{8 \mu}{h^{3}} a^{3}
$$

where $h$ is the separation of the capacitor plates, $a$ is the radius of the capacitor plates, and $\mu$ is the viscosity of air. The damping force is strongly dependent on the capacitor plate radius and the gap, thus to reduce the damping force, an array of small holes was machined into the capacitor plate by excimer laser machining.

The open-loop step response of the resonator was calculated using Matlab. The open-loop settling time (in ambient air) was less than $10 \mathrm{~ms}$ for a $10-\mu \mathrm{m}$ displacement (frequency step from $2.5 \mathrm{GHz}$ to $1.5 \mathrm{GHz}$ ), and the maximum sweep rate of the device was $10 \mu \mathrm{m} / \mathrm{ms}$, corresponding to a continuous frequency sweep rate of $1 \mathrm{GHz} / \mathrm{ms}$.

\section{Electrical Testing Results}

The tunable resonator had a resonant $Q$ of up to 380 at $2.5 \mathrm{GHz}$, although this measurement was conservative as it did not account for the insertion loss of the sub-miniature version A (SMA) connectors on the input and output ports (approximately $0.1 \mathrm{~dB}$ each). Fig. 4 shows measured $S_{21}$ for a range of tuning voltages. Out-of-band isolation was better than $-30 \mathrm{~dB}$ up to $4 \mathrm{GHz}$. 


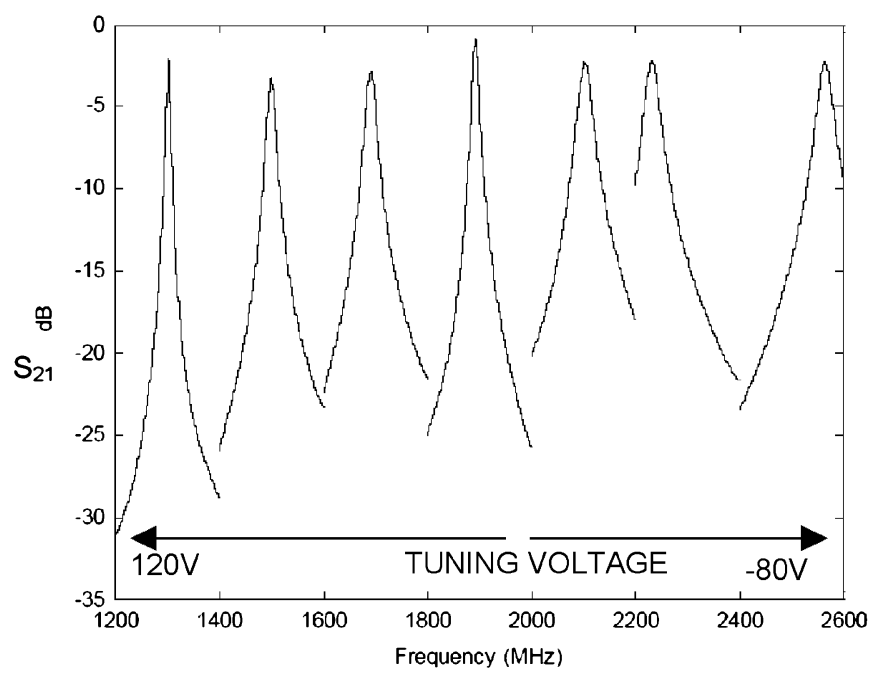

Fig. 4. Typical $S_{21}$ responce for two-port $S$-band resonator, IL $<-2.0 \mathrm{~dB}$, center frequency swept from 1.3 to $2.6 \mathrm{GHz}$.

TF1328 Act. Spectrum Res. Osc at 2.0GHZ, HBT Amp, 6dB ATTEN

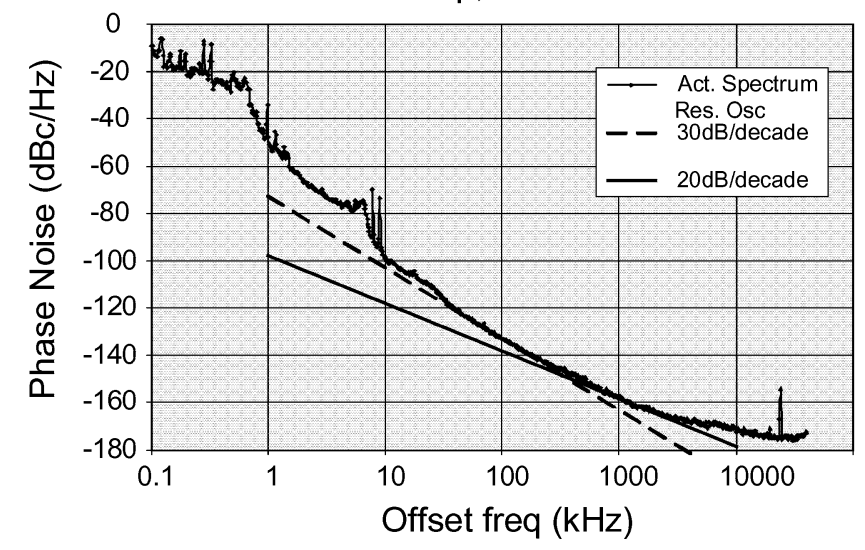

Fig. 5. Phase noise of oscillator loaded by two-port resonator.

A typical plot of phase noise versus offset frequency, Fig. 5, shows phase noise of $-132 \mathrm{dBc} / \mathrm{Hz}$ at $100-\mathrm{KHz}$ offset, at $2.0 \mathrm{GHz}$.

The third-order intermodulation was measured to have an IP3 intercept at $+41 \mathrm{dBm}$ for a $+10-\mathrm{dBm}$ carrier power and $5.3-\mathrm{kHz}$ offset [18]. For larger frequency offset the IP3 intercept rises to above $+47 \mathrm{dBm}$ (the limit of the sensitivity of the measurement apparatus). The peak in the intermodulation products at $5.3-\mathrm{kHz}$ frequency offset is a consequence of the mechanical self-excitation of the device at high power levels. No degradation of the resonator response was observed at input power levels of up to $+37 \mathrm{dBm}$ (the limit of the amplifiers in the test setup), although heating caused some drift of the resonator center frequency.

\section{CONCLUSION}

Microphonics must be addressed for commercial-production devices, as well as sensitivity to external vibrations and self-excitation at high power. Further work in optimizing the thermal response of the resonator is also necessary for the device to be useable in harsh environments. To address these shortcomings, a feedback controller will be built into the positioning system, in addition to commercially-available packaging refinements (for example a hermetic vacuum package) and a more judicious materials selection to minimize CTE mismatch between the electroformed cavity and the ceramic substrate.

\section{ACKNOWLEDGMENT}

The authors wish to thank Dr. D. Ham, Harvard University, for assistance with the microwave testing.

\section{REFERENCES}

[1] L. R. Whicker, Ferrite Control Components: Junction Circulators, YIG Filters and Limiters. Boston, MA: Artech, 1974.

[2] L. Sengupta and S. Sengupta, "Novel ferroelectric materials for phased array antennas," IEEE Trans. Ultrason., Ferroelect. Freq. Contr., vol. 44, no. 4, pp. 792-797, Jul. 1997.

[3] J. Breitbarth, "Octave tuning, high frequency varactor oscillator design," M.S. thesis, Univ. Colorado, Boulder, 2001.

[4] A. Brown and G. Rebeiz, "A varactor-tuned RF filter," IEEE Trans. Microw. Theory Tech., vol. 48, no. 7, pp. 1157-1160, Jul. 2000.

[5] D. Harris, "A 4-40 GHz wide bandwidth, magnetically tuned bandpass filter," in IEEE MTT-S Dig., 1990, pp. 1019-1022.

[6] D. Oates and G. Dionne, "Magnetically tunable superconducting resonators and filters," IEEE Trans. Appl. Supercond., vol. 9, no. 2, pp. 4170-4175, Jun. 1999.

[7] R. T. Chen et al., "A compact low-loss ka-band filter using 3-dimensional micromachined integrated coax," in Proc. 17th IEEE Int. Conf. (MEMS'04), Jan. 2004, pp. 801-804.

[8] G. Rebeiz, RF MEMS Theory, Design and Technology. New York: Wiley, 2003.

[9] X. Gong, A. Margomenos, B. Liu, S. Hajela, W. J. Chappell, and L. P. B. Katehi, "Precision fabrication techniques and analysis on high-Q evanescent-mode resonators and filters of different geometries," IEEE Trans. Microw. Theory Tech., vol. 52, no. 11, pp. 2557-2566, Nov. 2004

[10] A. D. Margomenos, "Three dimensional integration and packaging using silicon micromachining," Ph.D. dissertation, Univ. Michigan, Ann Arbor, 2004.

[11] T. George, "Overview of MEMS/NEMS Technology Development for Space Applications at NASA/JPL," Tech. Rep., [Online] Available: http://mems.jpl.nasa.gov, 2003.

[12] A. Slocum, Precision Machine Design. Englewood Cliffs, NJ: Prentice-Hall, 1992.

[13] J. White, "The nanogate: nanoscale flow control," Ph.D. dissertation, Mass. Inst. Technol., Cambridge, 2003.

[14] G. Binning and H. Rohrer, "Scanning tunneling microscopy," IBM. J. Res. Develop., vol. 30, no. N4 355, 1986.

[15] S. Hou, J. Lang, A. Slocum, A. Weber, and J. White, "A high-Q widelytunable gigahertz electromagnetic cavity resonator," in Proc. Solid State Sensor, Actuator Microsystems Workshop, Hilton Head, SC, Jun. 6-10, 2004.

[16] E. Garcia and N. Lobontiu, "Induced-strain multimorphs for microscale sensory actuation design," Smart Mater. Struct., vol. 13, pp. 725-732, 2004.

[17] J. J. Blech, "On isothermal squeeze films," J. Lubrication Technol., vol. 105, pp. 615-620, 1983.

[18] B. Myers, Measuring IP3, Agilent Technologies Design Tip, 2005. 